\title{
Inhibition of Lipocalin 2 Impairs Breast Tumorigenesis and Metastasis
}

\author{
Xiaohong Leng, ${ }^{1}$ Tian Ding, ${ }^{1}$ Hui Lin, ${ }^{1}$ Yan Wang, ${ }^{1}$ Limei Hu, ${ }^{2}$ Jianhua Hu, ${ }^{3}$ Barry Feig, ${ }^{4}$ \\ Wei Zhang, ${ }^{2}$ Lajos Pusztai, ${ }^{5}$ W. Fraser Symmans, ${ }^{2}$ Yun $W u,{ }^{2}$ and Ralph B. Arlinghaus ${ }^{1}$
}

Departments of ${ }^{1}$ Molecular Pathology, ${ }^{2}$ Pathology, ${ }^{3}$ Biostatistics, ${ }^{4}$ Surgical Oncology, and ${ }^{5}$ Breast Medical Oncology, The University of Texas M. D. Anderson Cancer Center, Houston, Texas

\begin{abstract}
Lipocalin 2 (LCN2; also known as NGAL) is a secreted glycoprotein and its elevated expression has been observed in breast cancers. However, the importance of LCN2 in breast tumorigenesis is unclear. Here, we employed a spontaneous mammary tumor mouse model showing that MMTV-ErbB2 (V664E) mice lacking mouse LCN2 had significantly delayed mammary tumor formation and metastasis with reduced matrix metalloproteinase-9 activity in the blood. LCN2 expression is upregulated by HER2/phosphoinositide 3-kinase/ AKT/NF- $\kappa$ B pathway. Decreasing LCN2 expression significantly reduced the invasion and migration ability of $\mathrm{HER2}^{+}$breast cancer cells. Furthermore, injecting an anti-mouse LCN2 antibody into mice bearing established murine breast tumors resulted in significant blockage of lung metastasis. Our findings indicate that LCN2 is a critical factor in enhancing breast tumor formation and progression possibly in part by stabilizing matrix metalloproteinase-9. Our results suggest that inhibition of LCN2 function by an inhibitory monoclonal antibody has potential for breast cancer therapy, particularly by interfering with metastasis in aggressive types of breast cancer. [Cancer Res 2009;69(22):8579-84]
\end{abstract}

\section{Introduction}

Recent studies have implicated lipocalin 2 (LCN2), a member of the lipocalin family, in leukemia (1-3) and other solid tumors (4). The lipocalin family is a large group of small secreted glycoproteins involved in binding and transportation of small lipophilic molecules (5). The physiologic ligand for LCN2 is not known. On bacterial infection, LCN2 tightly binds to bacterial iron-siderophores and deprives the iron source for their growth (6), which is shown by the delayed clearance of bacteria infection in LCN2 knockout mice $(7,8)$.

It was observed that increased LCN2 levels were present in the plasma of leukemia patients with CML (1-3). We found that mouse LCN2 (mLCN2) is required for BCR-ABL-induced leukemia in a mouse bone marrow transplant model and $\mathrm{BCR}-\mathrm{ABL}^{+}$mouse leukemic cells use the secreted LCN2 to reduce hematopoietic cells in normal bone marrow and spleen by inducing apoptosis of normal

Note: Supplementary data for this article are available at Cancer Research Online (http://cancerres.aacrjournals.org/).

X. Leng and T. Ding contributed equally to this work. Y. Wu and R.B. Arlinghaus are co-corresponding authors.

Requests for reprints: Ralph B. Arlinghaus, Department of Molecular Pathology, The University of Texas M. D. Anderson Cancer Center, Unit 951, 7435 Fannin Street Houston, TX 77054. Phone: 713-792-8995; Fax: 713-834-6082; E-mail: rarlingh@mdanderson. org and Yun Wu, Department of Pathology, The University of Texas M. D. Anderson Cancer Center, Unit 085, 1515 Holcombe Boulevard, Houston, TX 77030. Phone: 713-563-1847; Fax 713-563-1848; E-mail: YunWu@mdanderson.org.

(C)2009 American Association for Cancer Research.

doi:10.1158/0008-5472.CAN-09-1934 hematopoietic cells for leukemia cell expansion $(1,3)$. The requirement of LCN2 for leukemia cell expansion is consistent with the previously identified role of LCN2 in inducing apoptosis of mouse hematopoietic cells on withdrawal of the cytokine growth factor interleukin-3 (9). The ability of LCN2 to mediate apoptosis appears to be due to its ability to export a still unknown iron-form from the target cells (10). Importantly, we found that LCN2 is also essential for solid tumor formation, as BCR-ABL-expressing leukemia cells lacking LCN2 expression failed to form s.c. allograft tumor nodules (3).

In breast cancer, a high molecular weight of complex consisting of LCN2 and matrix metalloproteinase-9 (MMP-9) was observed in the patient urine samples (11). Elevated LCN2 expression was shown to associate with decreased survival in breast cancer patients (12). Increased LCN2 expression was found to associate with increased tumor cell motility $(13,14)$. LCN2 was shown to stabilize MMP-9, a critical enzymatic activity required for extracellular matrix remodeling during tumor growth and metastasis $(15,16)$ and LCN2 may promote breast cancer progression by regulating epithelial-to-mesenchymal transition (14). However, the significance and how LCN2 is involved in breast carcinogenesis remain elusive.

We tested the role of LCN2 in breast tumorigenesis using a spontaneous MMTV-ErbB2(V664E) transgenic mammary tumor mouse model crossed with $\mathrm{LCN} 2^{-/-}$mice. Our findings presented here illustrate the importance of LCN2 in breast cancer tumor formation and metastasis and the possible underlying molecular mechanisms promoted by LCN2 expression in breast cancer, indicating that inhibition of LCN2 has potential in breast cancer therapy.

\section{Materials and Methods}

Tissue culture and mice. Cell lines were grown in DMEM with $10 \%$ fetal bovine serum and penicillin/streptomycin at $37^{\circ} \mathrm{C}$ in humidified incubator with $5 \% \mathrm{CO}_{2}$. LCN2 knockout $\left(\mathrm{LCN}^{-/-}\right)$mice were a gift from Dr. Alan Aderem (8). MMTV-ErbB2(V664E) transgenic mice (17) and athymic nude mice (8-12 weeks) were purchased from The Jackson Laboratory and Harlan, respectively. Mice experiments were done in The University of Texas M. D. Anderson Cancer Center Animal Facility with approved Institutional Animal Care and Use Committee protocols.

Antibodies and constructs. Purified polyclonal antibodies against mLCN2 or human LCN2 were generated in our laboratory using recombinant proteins purified from Escherichia coli, a gift from Dr. Roland Strong (6). Antibodies against AKT, pAKT, HER2, pY-HER2, and $\beta$-actin were purchased from Abcam. Dominant-negative and constitutively active AKT constructs are gifts from Dr. Zhou Songyang.

Short hairpin RNA experiments and lentiviral infection. Short hairpin RNA (shRNA) lentiviral constructs (Sigma-Aldrich) containing shRNAs for NGAL (clone D: TRCN0000060290 and clone E: TRCN0000060290) and $\mathrm{m} L C N 2$ (TRCN0000055328) were used. Puromycin was used to select cells containing shRNA after lentiviral infection.

Reverse transcription-PCR. Total RNA extracted from cells was used to synthesize the cDNA as PCR template. Reverse transcription-PCR 
primers for $\mathrm{mLCN2}$ : $5^{\prime}$-AGCCAGACTTCCGGAGCGATC-3' and 5'-ACTTGGCAAAGCGGGTGAAACG-3' and for mouse actin: 5'-GCTGGAAGGTGGACAGTGAG- $3^{\prime}$ and $5^{\prime}$-ATGGATGACGATATCGCTGC-3'.

Two-chamber migration and invasion assays. Cell migration and invasion assays were done using the two-chamber migration assay $(8 \mu \mathrm{m}$ pore size; BD Biosciences). For SKBr3 cells, $1 \times 10^{5}$ cells were seeded in serum-free medium in the upper chamber and migrated toward $10 \%$ FCS in the lower chamber for $18 \mathrm{~h}$ followed by fixation and staining with $0.2 \%$ crystal violet-20\% methanol. Quantification was done using unpaired $t$ test.

Western blotting and zymogram of plasma samples. Plasma (1-2 $\mu \mathrm{L})$ was separated on $20 \%$ SDS-PAGE followed by transferring and blotting with purified anti-mLCN2 polyclonal antibody (1:3,000). Plasma (2-5 $\mu \mathrm{L})$ was analyzed using $10 \%$ zymographic gel (Invitrogen).

Imaging, histology, H\&E staining, and immunohistochemistry. Freshly collected lung tissues were imaged using Xenogen IVIS 200 Imaging System for green fluorescent protein signals. For histologic analysis, tissues were postfixed with $10 \%$ neutral buffered formalin, embedded in paraffin, sectioned at $4 \mu \mathrm{m}$, and stained with H\&E.

Statistical data analysis. The Kaplan-Meier method (log-rank test) was used to determine the survival curves of tumor formation in MMTV-ErbB2 (V664E) mice with various alleles of $\mathrm{m} L C N 2$. The log-rank test was used in the intergroup comparisons. The Wilcoxon rank-sum test was used to analyze the fluorescence imaging results of the in vivo antibody studies.

\section{Results}

Upregulation of LCN2 (NGAL) through HER2/neu signaling pathway in human breast cancer. We observed a significant clinical correlation of higher LCN2 expression with HER2 positivity among 318 newly diagnosed breast cancer patients, ${ }^{6}$ implying that LCN2 might be a downstream target of the HER2 signaling pathway. When blocking HER2 function with Herceptin in HER2 ${ }^{+}$ SKBr3 cells, we found that LCN2 expression was inhibited (Fig. 1A). LCN2 expression in SKBr3 cells was reduced in a dosedependent manner by the phosphoinositide 3-kinase inhibitor LY294002 and by Bay 11-7082, which specifically blocks I $\kappa$ B phosphorylation needed for NF- $\kappa \mathrm{B}$ activity (Fig. $1 B$ and $C$ ). Secretion of LCN2 into conditioned medium was also effectively inhibited under these conditions (Supplementary Fig. S1). In addition, overexpression of dominant-negative AKT led to reduced expression of LCN2, whereas overexpression of constitutively active AKT increased LCN2 levels (Fig. $1 D$ ). These results indicate a role of the HER2/phosphoinositide 3-kinase/AKT/NF- $\kappa$ B signal cascade in inducing LCN2 expression in human breast cancer, consistent with a recent study showing that LCN2 expression was largely dependent on the NF- $\kappa \mathrm{B}$ pathway in thyroid neoplastic cells (18).

Mice lacking mLCN2 have impaired ErbB2(V664E)-induced mammary tumor formation. We tested mLCN2 effects in a spontaneous mouse mammary tumor model. Transgenic mice carrying the mutant form of ErbB2(V664E) driven by the mammary-specific promoter MMTV develop multiple primary breast tumors and lung metastases (17). By breeding MMTV-ErbB2(V664E) ${ }^{\mathrm{tg} / \mathrm{tg}}$ mice (FVB) and $\mathrm{mLCN} 2^{-/-}$mice (C57BL/6; ref. 8), we generated three groups of mice expressing ErbB2(V664E) with variations in $\mathrm{m} L C N 2$ alleles $\left(\mathrm{mLCN} 2^{+/+}, \mathrm{mLCN} 2^{+/-}\right.$, and $\mathrm{mLCN} 2^{-/-}$; Supplementary Fig. S2A-C).

In this genetic study for the effects of mLCN2 on ErbB2(V664E)induced breast tumors, we observed striking differences in the timing of tumor formation as well as the number and the size of primary tumors among the three groups. Groups carrying either one or two alleles of $\mathrm{mLCN} 2$ started to develop multiple large $(>1 \mathrm{~cm})$ mammary tumors $\sim 170$ days after birth. In contrast,

\footnotetext{
${ }^{6}$ Unpublished data.
}

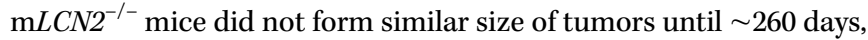
a time when $>60 \%$ mice in the groups expressing mLCN2 were already terminated due to excessive tumor burden (Fig. $2 A$ ). The overall tumor occurrence was significantly delayed in the $\mathrm{m} L C N 2^{-/-}$group (260-500 days with $T_{50}=303$ days) compared with the $\mathrm{mLCN2} 2^{+/+}$mice (170-340 days with $T_{50}=210$ days; Fig. $2 A$ ). Although the $\mathrm{mLCN} 2^{+/-}$group showed a slightly delayed course of tumor occurrence compared with the $\mathrm{m} L C N 2^{+/+}$group, no significance was observed regarding the tumor occurrence and the tumor volume between these two groups (Fig. $2 A$ and $B$ ), indicating that one allele $\mathrm{m} L C N 2$ deficiency was not sufficient to interfere with the formation of ErbB2(V644E)-induced breast tumors. We observed greater numbers and larger volumes of tumors per mouse in the groups expressing mLCN2 compared with the $\mathrm{mLCN} 2^{-/-}$group (Fig. 2B; Supplementary Fig. S2D). Notably, the lung metastases in $\mathrm{m} L C N 2^{-/-}$mice were significantly delayed compared with the $\mathrm{mLCN} 2^{+/+}$mice $(P<0.05$; Fig. $2 C)$. By Kaplan-Meier analysis, the $T_{50}$ for lung metastasis in the $\mathrm{mLCN} 2^{+/+}$group is $\sim 260$ days. In contrast, the $T_{50}$ value was not reached in the $\mathrm{mLCN2^{+/- }}$ and the $\mathrm{mLCN2} 2^{-/-}$ groups, suggesting that deficient $\mathrm{m} L C N 2$ expression also impairs lung metastasis in this model.

Unlike the healthy wild-type mice with minimal levels of mLCN2 in the plasma (Fig. 2D, top, last three lanes), we detected a dramatically increased mLCN2 level in the plasma of tumor-bearing MMTV-ErbB2(V664E) mice (Fig. 2D, top). Interestingly, we observed elevated MMP-9 gelatinase activity and the presence of higher molecular weight gelatinase activity in the plasma of tumor-bearing mice expressing mLCN2 compared with $\mathrm{mLCN} 2^{-/-}$ group (Fig. 2D, bottom, outlined with red box). We also noticed the relatively weak MMP-9 gelatinase activity in the plasma of tumorbearing $\mathrm{mLCN} 2^{-/-}$mice (Fig. $2 D$, bottom), and these samples displayed distinct gelatinase bands with molecular weight lower than the MMP-9 band, suggesting the beneficial effects of mLCN2 in maintaining MMP-9 activity potentially though stabilizing MMP-9 as implied by the previous studies $(15,16)$.

LCN2 expression in $\mathrm{HER2}^{+}$breast tumor cells stimulates cell invasion in vitro and their metastatic potential in mouse xenograft model. Using HER2 ${ }^{+}$SKBr3 cells (high LCN2 expression),

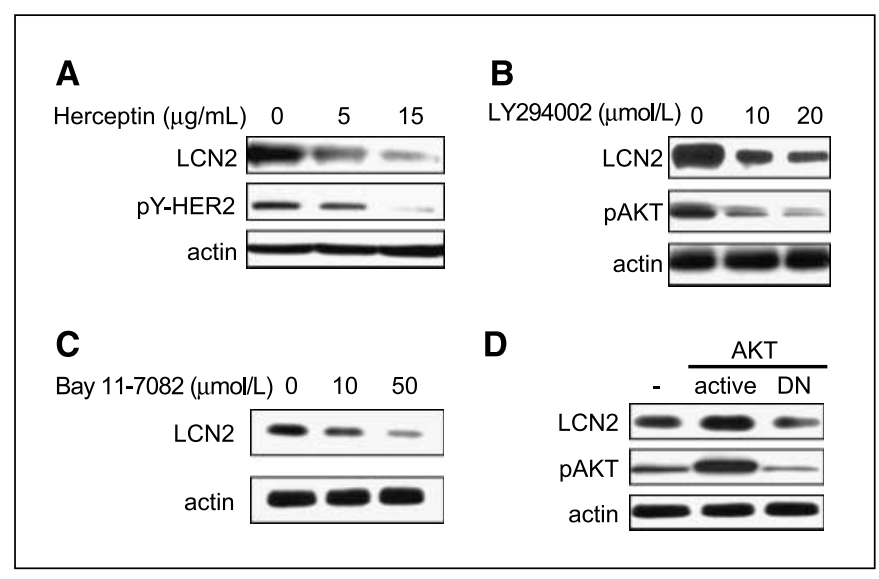

Figure 1. LCN2 (NGAL) is a downstream target of HER2/phosphoinositide 3-kinase/AKT/NF-KB pathway. $A$, Western blotting of LCN2 levels in HER2 ${ }^{+}$ SKBr3 cells treated with Herceptin. B, Western blotting of LCN2 levels in SKBr3 cells incubated with the phosphoinositide 3-kinase inhibitor LY294002. C Western blotting of LCN2 levels in SKBr3 cells incubated with the NF-KB inhibitor Bay 11-7082. D, Western blotting of LCN2 levels in SKBr3 cells transiently expressing either constitutively active or dominant-negative (DN) AKT. 
A

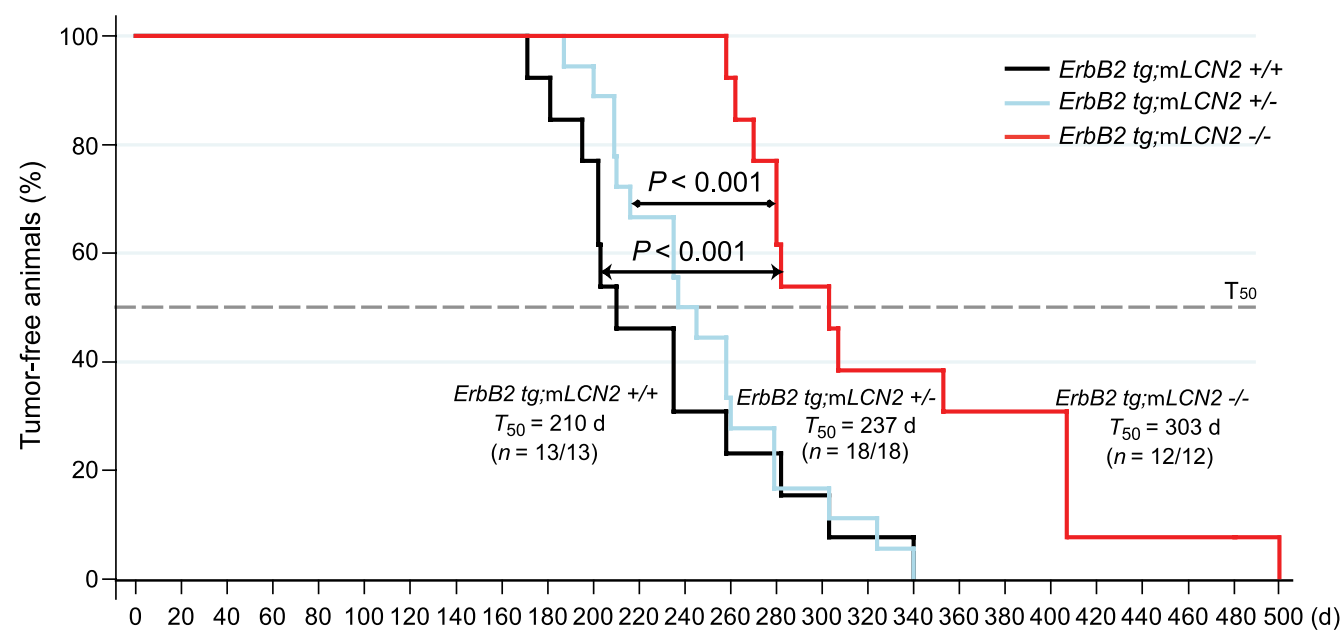

B

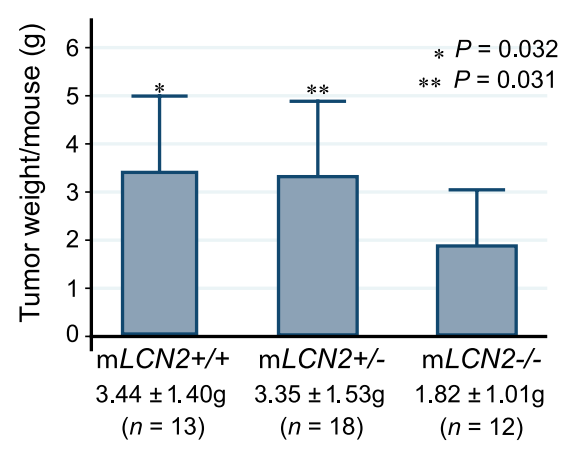

C

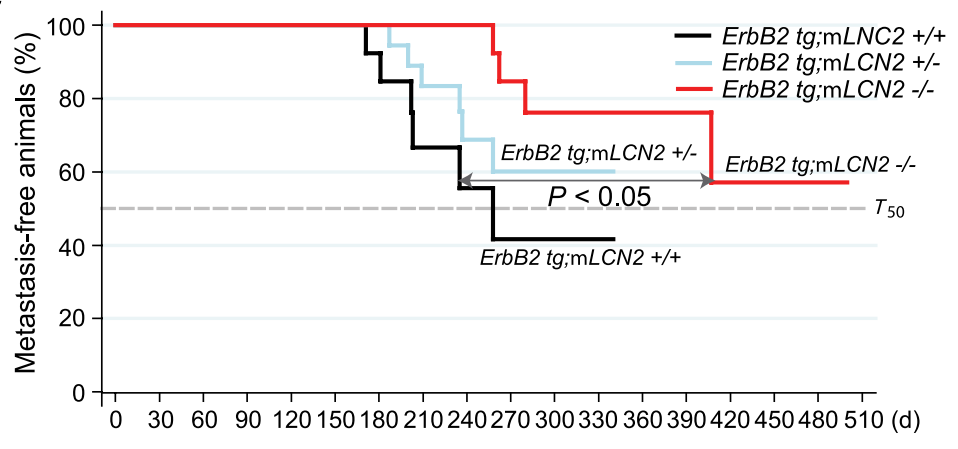

D

plasma

$\mathrm{mLCN} 2 \rightarrow$

$\mathrm{mLCN} 2+/+$

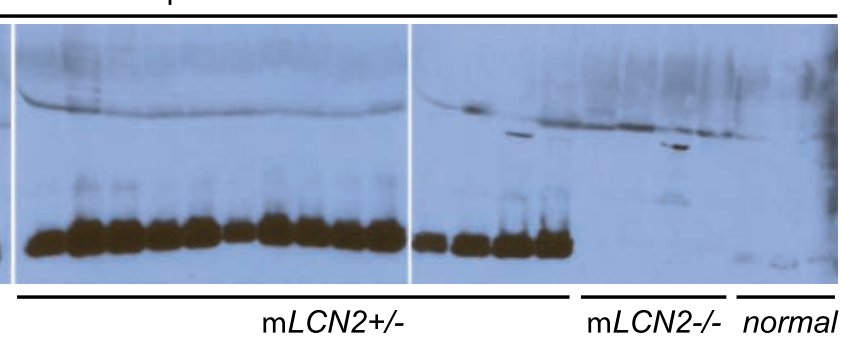

$\mathrm{mLCN} 2+/-$

MMP-9

transfection

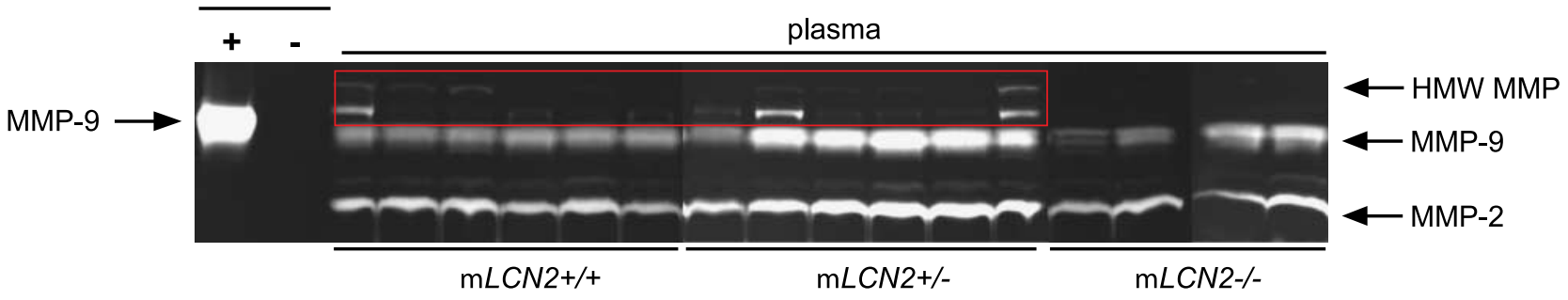

Figure 2. Effects of $\mathrm{mLCN} 2$ on the mammary tumor formation and metastasis in the MMTV-ErbB2(V664E) transgenic mouse model. A, time course of primary mammary tumor development in MMTV-ErbB2(V664E) transgenic mice in the three genetic backgrounds: $\mathrm{mLCN2} 2^{++}, \mathrm{mLCN2} 2^{+/-}$, and $\mathrm{mLCN2}{ }^{-/-}$, depicted by the Kaplan-Meier analysis. $T_{50}$ is a calculated statistical value incorporating both time and incidence of tumor formation when $50 \%$ of the mice in the same group developed mammary tumors. $B$, summary of total breast tumor weights per mouse in the three mice groups. $C$, Kaplan-Meier analysis of lung metastasis of the three mice groups. $D$, levels of $\mathrm{mLCN} 2(t o p)$ in the plasma of the three mice groups at euthanization. $\mathrm{mLCN} 2$ levels in the plasma from normal healthy mice were shown in the last three lanes. MMP activity in the plasma of the three mice groups was analyzed using $10 \%$ zymogram gel (bottom). Conditioned medium from MMP-9-transfected 293T cells was used to mark MMP-9 position. Red box outlined the high molecular weight of MMP activity (HMW MMP). 
we observed a significant reduction in cell migration and invasion on knocking down LCN2 expression compared with either parental cells or cells expressing nonspecific shRNA (Fig. $3 A$ and $B$ ). We used MDA-MB-468 (high LCN2 expression) to test LCN2 effects in mouse xenograft model. Note, we did not observe any change in cell growth rate in culture on lowering LCN2 expression by shRNA in either SKBr3 or MDA-MB-468 cells (data not shown; Supplementary Fig. S $3 A$ ). We injected mammary fat pads of nude mice with 1 million of either parental MDA-MB-468 cells or its derivatives expressing either the nontargeted shRNA or the shRNA for LCN2. We analyzed the primary tumor and the surrounding tissues 42 days after implantation. No significant differences in the primary tumor size/weight were found among the three groups (Supplementary Fig. S3B). However, the capacity of tumor cells for invasion and metastasis, as measured by the events of lymphovascular invasion (Fig. 3C, b), intramammary lymph node metastasis (Fig. $3 C, c$ ), and chest/abdominal wall invasion (Fig. $3 C, d$ ), were significantly reduced in the group injected of MDA-MB-468 cells with the LCN2 shRNA knockdown (Fig. 3D).

mLCN2 expression correlates with aggressive tumor formation in murine mammary tumor cell lines. The association of LCN2 expression with aggressive human breast cancer types was further shown when using a series of mouse breast tumor cell lines (67NR, 168FARN, 4T07, and 4T1), which were derived from the mammary tumors of the same mouse but with distinct metastatic potentials (19). 4T07 and 4T1 cells are the most aggressive and develop lung metastases. We found that only 4T07 and 4T1 cells have $\mathrm{mLCN} 2$ transcripts and secrete $\mathrm{mLCN} 2$, with the most aggressive 4T1 cells having the highest levels of mLCN2 (Fig. 4A). Knocking down $\mathrm{m} L C N 2$ by shRNA in 4T1 cells (Supplementary Fig. S4A, left) reduced MMP-9 activity (Supplementary Fig. S4A, right). Reduction of mLCN2 expression greatly decreased 4T1 invasive ability (Supplementary Fig. S4B) and colony formation in soft agar (Supplementary Fig. $\mathrm{S} 4 C$ ). Similar to our findings in the plasma from

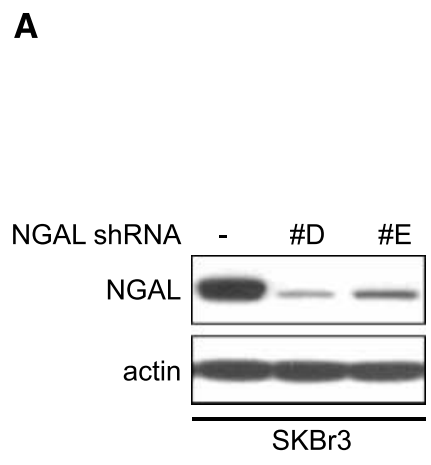

B
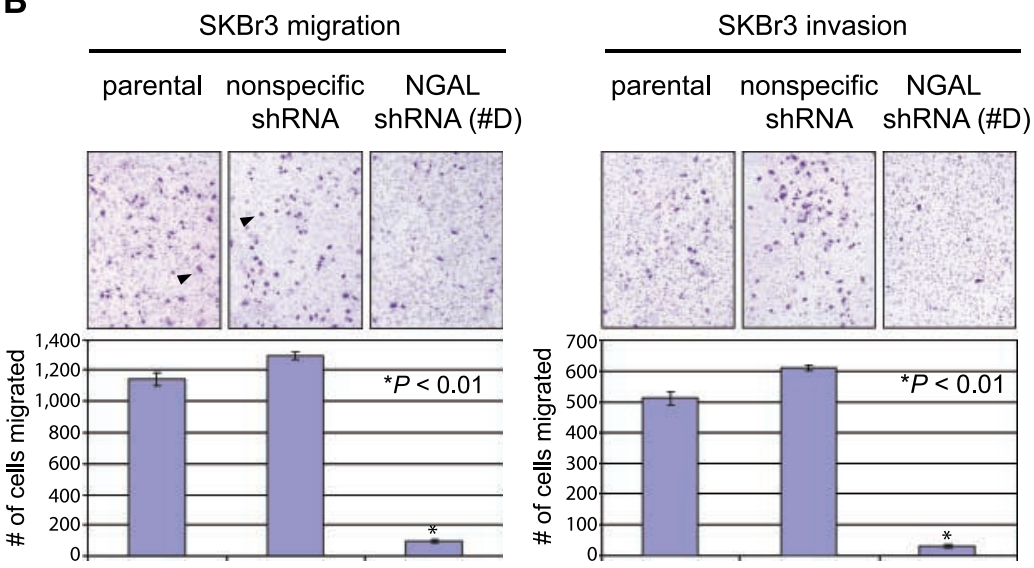

C
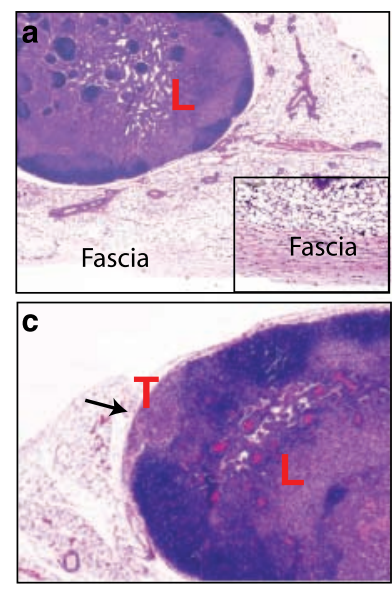
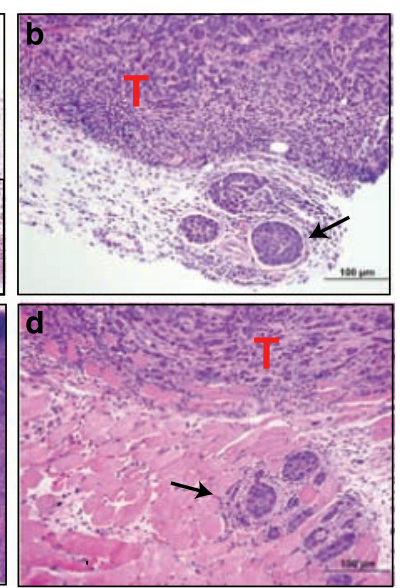

D

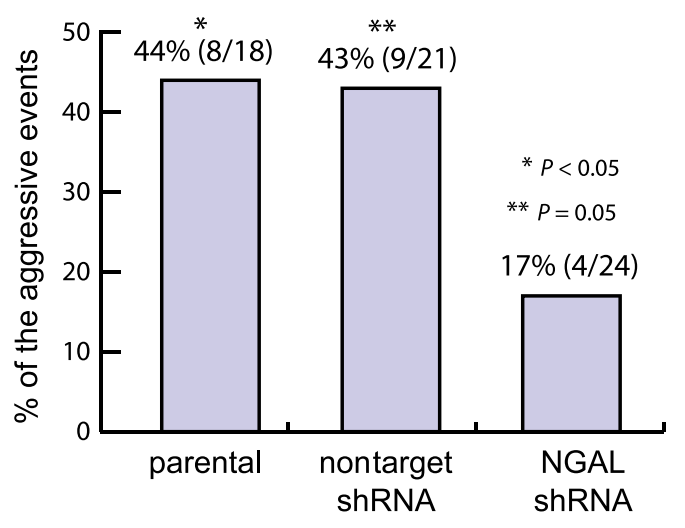

Figure 3. LCN2 (NGAL) expression stimulates cell invasion and metastasis of human breast cancer cells. $A$, Western blotting of NGAL levels in SKBr3 with

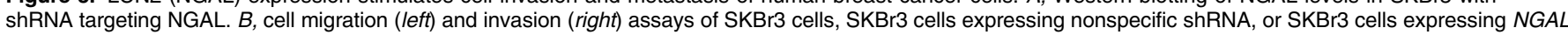
shRNA. $C, \mathrm{H} \& \mathrm{E}$ staining of the fourth mammary gland from normal mouse (a) showing normal intramammary lymph node structure and fascia as a barrier to block

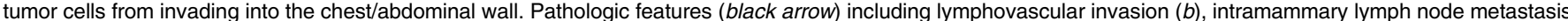

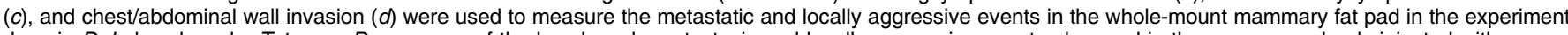
done in $D$. L, lymph node; $T$, tumor. $D$, summary of the lymph node metastasis and locally aggressive events observed in the mammary glands injected with MDA-MB-468 cells or MDA-MB-468 cells expressing either nontargeted shRNA or NGAL shRNA. Statistical analysis was done with Pearson's $\mathrm{X}^{2}$ test. 
A

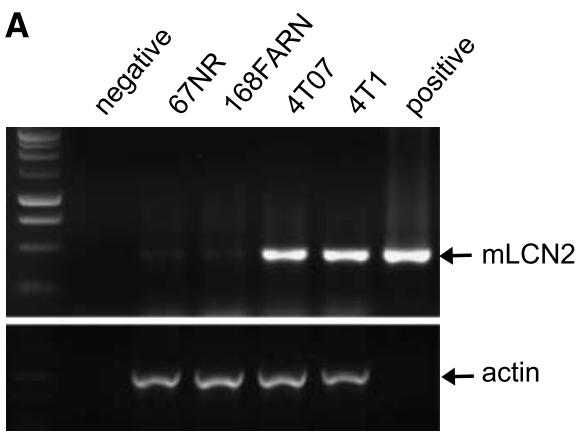

RT-PCR

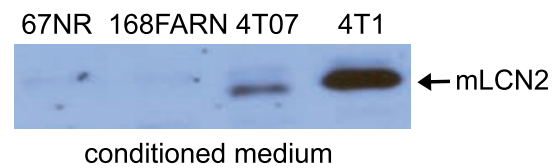

C

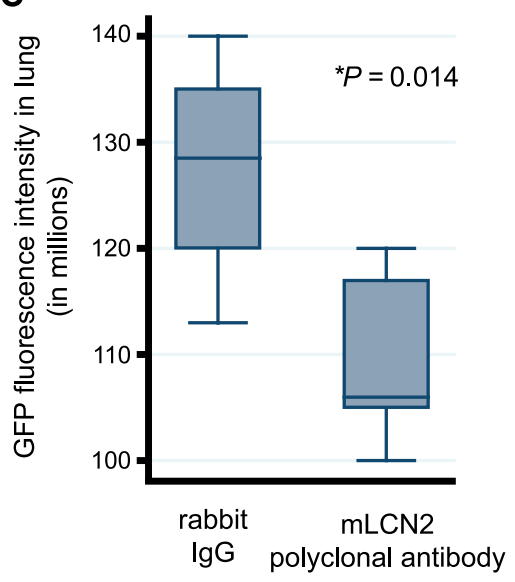

B

Treated with rabbit IgG

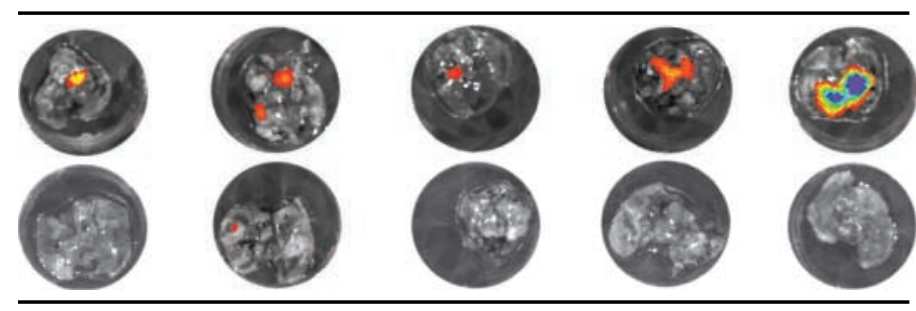

Treated with $\mathrm{mLCN} 2$ polyclonal antibody

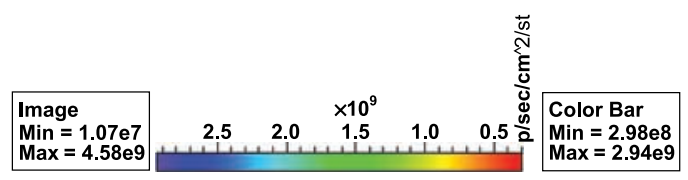

D

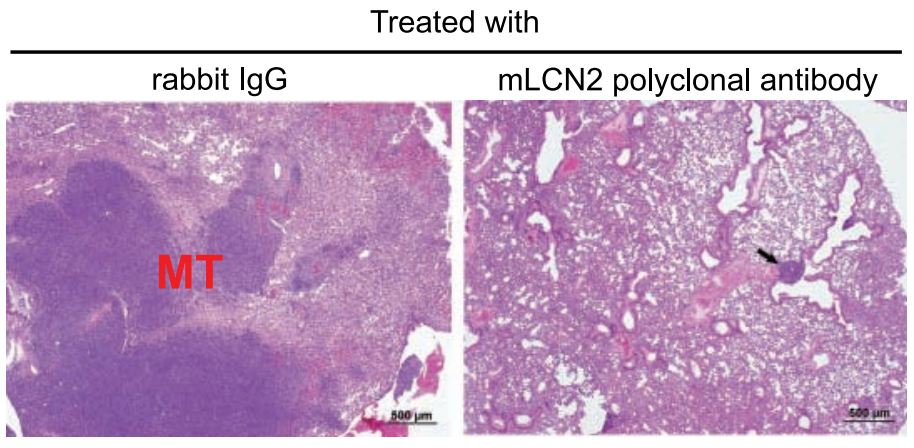

Figure 4. Reduction of lung metastasis by $\mathrm{mLCN} 2$ antibody injection in the $4 \mathrm{~T} 1$ breast cancer model. $A$, expression of $\mathrm{mLCN} 2$ is associated with aggressive mouse mammary tumor cell lines. Top, reverse transcription-PCR of mLCN2 transcripts in 67NR, 168FARN, 4T07, and 4T1 cells: actin as internal control and a plasmid containing $\mathrm{mLCN} 2$ as positive control. Bottom, Western blotting of $\mathrm{mLCN} 2$ levels in the conditioned medium of the four cell lines. $B$, in vivo metastasis assay of 4T1 cells of mice injected with purified rabbit IgG (top) or purified rabbit anti-mLCN2 polyclonal antibody (bottom). Lung metastasis burden was monitored using Xenogen IVIS 200 Imaging System. The color scale depicts the green fluorescent protein signal emitted from the metastatic $4 \mathrm{~T} 1$ cells. $C$, comparison of total green fluorescent protein signal intensity in either the control antibody-treated group or the anti-mLCN2 antibody-treated group. $D$, representative H\&E staining of lung metastasis of $4 \mathrm{~T} 1$ tumor-bearing mice treated with purified rabbit IgG (left) or purified anti-mLCN2 antibody (right). MT, metastatic tumor. Black arrow, small metastatic tumor lesion.

ErbB2-induced breast tumor-bearing mice, we detected high levels of mLCN2 in the plasma of breast tumor-bearing mice implanted with 4T1 cells and increased MMP-9 activity in the plasma compared with normal healthy mice (Supplementary Fig. S4D).

I.v. administration of anti-mLCN2 antibody to mice with established mammary tumors reduces lung metastases. To explore the possibility that inhibition of the secreted LCN2 might block distant metastasis, we i.v. injected an affinity-purified rabbit polyclonal antibody against mLCN2 (anti-mLCN2) into nude mice 7 days after implantation with 5,000 green fluorescent proteinlabeled $4 \mathrm{~T} 1$ cells, when visible breast tumors $(\sim 2 \mathrm{~mm})$ were formed. Antibody injection $(\sim 100 \mu \mathrm{g})$ was done once per week for four times, with purified rabbit IgG as control. We observed a dramatic decrease in lung metastases in the anti-mLCN2 antibodytreated group compared with the control IgG-treated group as measured by green fluorescent protein signal intensities in freshly collected lung tissues $(P=0.028$; Fig. $4 B$ and $C$; Supplementary Fig.
S5A). Histologic examination confirmed significant differences in lung metastases between the two groups (Fig. $4 D$ ). We also found that the levels of circulating $4 \mathrm{~T} 1$ tumor cells were reduced in the blood of anti-mLCN2 antibody-treated mice (Supplementary Fig. S5B), suggesting that the antibody was able to block the emergence of disseminating tumor cells from the primary tumor sites. Taken together, our results strongly suggest that LCN2 could serve as a new therapeutic target for treating breast cancer metastasis.

\section{Discussion}

The results presented here provide strong preclinical and experimental evidence that LCN2 is a critical factor that facilitates breast tumorigenesis and metastasis, and it is a potential therapeutic target for aggressive forms of breast cancer. We were the first to use a spontaneous mouse breast cancer model to study the function of LCN2 in breast tumorigenesis. Mice that lack mLCN2 
expression had a significant delay in the formation of breast tumors in the MMTV-ErbB2(V664E) ${ }^{\mathrm{tg}}$ background, and when formed, these tumors displayed reduced tumor numbers and size compared with that from $\mathrm{mLCN} 2^{+/+}$and $\mathrm{mLCN} 2^{+/-}$mice. The timing of lung metastases was correspondingly delayed in the $\mathrm{m} L C N 2^{-/-}$ mice compared with the $\mathrm{mLCN2} 2^{+/+}$mice. This result indicates that although breast tumor formation and metastasis may not absolutely require LCN2 expression, LCN2 plays an essential role in enhancing the aggressiveness of breast cancer formation and metastasis. Our studies using human breast cancer cell lines and murine mammary tumor cells indicate that LCN2 expression led to more aggressive behavior including lung metastasis, tumor cell migration and invasion, and anchorage-independent growth in vitro.

How LCN2 is involved in solid tumor formation and progression is not fully understood. We observed an overall increased MMP-9 gelatinase activity, formation of higher molecular weight MMP complexes (20), and increased MMP-9 stability in tumor-bearing mice expressing LCN2. In contrast, in tumor-bearing mice with ablated LCN2 expression, an overall decrease in higher molecular weight MMP activities and MMP-9 activity was observed, consistent with the previous findings of the protective role of LCN2 on MMP-9 (16). The importance of these higher MMP activities in tumorigenesis is not known. The effects of loss of LCN2 expression on these MMP-related events are not yet understood and need further investigation.

Most importantly, we provided evidence that i.v. injection of an affinity-purified antibody made against mLCN2 strongly interfered with lung metastasis in an aggressive mouse 4T1-induced mammary tumor model. The primary breast tumors were not prevented from continued growth by i.v. injection of the mLCN2 antibody, which may be due to the insufficient levels of the mLCN2 antibody in reaching an already established tumor microenvironment. However, we did observe that anti-mLCN2 antibody-treated mice had slightly smaller primary tumors (10\% reduction on average; data not shown). We found less 4T1 cells in the blood of anti-mLCN2 antibody-treated mice. Possible mechanisms include that the antimLCN2 destabilizes LCN2/MMP-9 complex, thereby reducing the exit of 4T1 cells from the primary tumor mass and decreasing distant tumor metastasis. More studies are needed to clarify the mechanism of how anti-LCN2 reduces metastases. Our findings suggest that blocking LCN2 in blood circulation using a neutralizing monoclonal antibody against LCN2 might be an effective therapeutic option to interfere with breast cancer metastasis either alone or in combination with current therapeutic approaches.

\section{Disclosure of Potential Conflicts of Interest}

No potential conflicts of interest were disclosed.

\section{Acknowledgments}

Received 5/27/09; revised 8/21/09; accepted 9/16/09; published OnlineFirst 11/3/09. Grant support: Susan G. Komen Foundation grant KG081319 (Y. Wu and R.B. Arlinghaus), Department of Defense grant BC076078 (R.B. Arlinghaus), and The University of Texas M. D. Anderson Cancer Center startup fund (Y. $\mathrm{Wu}$ ).

R.B. Arlinghaus is a holder of the Stringer Chair of Cancer Research.

The costs of publication of this article were defrayed in part by the payment of page charges. This article must therefore be hereby marked advertisement in accordance with 18 U.S.C. Section 1734 solely to indicate this fact.

We thank Dr. Alan Aderem for the LCN2 KO mice, Dr. Roland Strong for the recombinant LCN2 proteins, Dr. Zhou Songyang for the AKT expression constructs, and Drs. Yibing Deng and Sendurai Mani for discussions and suggestions of the data in the article.

\section{References}

1. Lin H, Monaco G, Sun T, et al. Bcr-Abl-mediated suppression of normal hematopoiesis in leukemia. Oncogene 2005;24:3246-56.

2. Villalva C, Sorel N, Bonnet ML, et al. Neutrophil gelatinase-associated lipocalin expression in chronic myeloid leukemia. Leuk Lymphoma 2008;49:984-8.

3. Leng X, Lin H, Ding T, et al. Lipocalin 2 is required for BCR-ABL-induced tumorigenesis. Oncogene 2008;27 6110-9.

4. Devarajan P. Neutrophil gelatinase-associated lipocalin: new paths for an old shuttle. Cancer Ther 2007;5: 463-70.

5. Flower DR, North AC, Sansom CE. The lipocalin protein family: structural and sequence overview. Biochim Biophys Acta 2000;1482:9-24.

6. Goetz DH, Holmes MA, Borregaard N, Bluhm ME, Raymond KN, Strong RK. The neutrophil lipocalin NGAL is a bacteriostatic agent that interferes with siderophore-mediated iron acquisition. Mol Cell 2002, 10:1033-43.

7. Berger T, Togawa A, Duncan GS, et al. Lipocalin 2-deficient mice exhibit increased sensitivity to Escherichia coli infection but not to ischemia-reperfusion injury. Proc Natl Acad Sci U S A 2006;103:1834-9.

8. Flo TH, Smith KD, Sato S, et al. Lipocalin 2 mediates an innate immune response to bacterial infection by sequestrating iron. Nature 2004;432:917-21.

9. Devireddy LR, Teodoro JG, Richard FA, Green MR. Induction of apoptosis by a secreted lipocalin that is transcriptionally regulated by IL-3 deprivation. Science 2001;293:829-34.

10. Devireddy LR, Gazin C, Zhu X, Green MR. A cellsurface receptor for lipocalin $24 \mathrm{p} 3$ selectively mediates apoptosis and iron uptake. Cell 2005;123:1293-305.

11. Fernandez CA, Yan L, Louis G, Yang J, Kutok JL, Moses MA. The matrix metalloproteinase-9/neutrophil gelatinase-associated lipocalin complex plays a role in breast tumor growth and is present in the urine of breast cancer patients. Clin Cancer Res 2005;11: 5390-5.

12. Bauer M, Eickhoff JC, Gould MN, Mundhenke C, Maass N, Friedl A. Neutrophil gelatinase-associated lipocalin (NGAL) is a predictor of poor prognosis in human primary breast cancer. Breast Cancer Res Treat 2008;108:389-97.

13. Hu L, Hittelman W, Lu T, et al. NGAL decreases Ecadherin-mediated cell-cell adhesion and increases cell motility and invasion through Rac1 in colon carcinoma cells. Lab Invest 2009;89:531-48.

14. Yang J, Bielenberg DR, Rodig SJ, et al. Lipocalin 2 promotes breast cancer progression. Proc Natl Acad Sci U S A 2009;106:3913-8.
15. Kubben FJ, Sier CF, Hawinkels LJ, et al. Clinical evidence for a protective role of lipocalin-2 against MMP-9 autodegradation and the impact for gastric cancer. Eur J Cancer 2007;43:1869-76.

16. Yan L, Borregaard N, Kjeldsen L, Moses MA. The high molecular weight urinary matrix metalloproteinase (MMP) activity is a complex of gelatinase B/MMP-9 and neutrophil gelatinase-associated lipocalin (NGAL). Modulation of MMP-9 activity by NGAL. J Biol Chem 2001;276:37258-65.

17. Muller WJ, Sinn E, Pattengale PK, Wallace R, Leder P. Single-step induction of mammary adenocarcinoma in transgenic mice bearing the activated c-neu oncogene. Cell 1988;54:105-15.

18. Iannetti A, Pacifico F, Acquaviva R, et al. The neutrophil gelatinase-associated lipocalin (NGAL), a NF- $\kappa$ B regulated gene, is a survival factor for thyroid neoplastic cells. Proc Natl Acad Sci U S A 2008;105:14058-63.

19. Aslakson CJ, Miller FR. Selective events in the metastatic process defined by analysis of the sequential dissemination of subpopulations of a mouse mammary tumor. Cancer Res 1992;52:1399-405.

20. Roy R, Louis G, Loughlin KR, et al. Tumor-specific urinary matrix metalloproteinase fingerprinting: identification of high molecular weight urinary matrix metalloproteinase species. Clin Cancer Res 2008;14: 6610-7. 


\section{Cancer Research}

\section{Inhibition of Lipocalin 2 Impairs Breast Tumorigenesis and Metastasis}

Xiaohong Leng, Tian Ding, Hui Lin, et al.

Cancer Res 2009;69:8579-8584. Published OnlineFirst November 3, 2009.

\section{Updated version Access the most recent version of this article at: doi:10.1158/0008-5472.CAN-09-1934}

Supplementary Access the most recent supplemental material at:

Material http://cancerres.aacrjournals.org/content/suppl/2009/11/03/69.22.8579.DC1 http://cancerres.aacrjournals.org/content/suppl/2009/11/03/0008-5472.CAN-09-1934.DC1

Cited articles This article cites 20 articles, 8 of which you can access for free at:

http://cancerres.aacrjournals.org/content/69/22/8579.full.html\#ref-list-1

Citing articles This article has been cited by 22 HighWire-hosted articles. Access the articles at: /content/69/22/8579.full.html\#related-urls

E-mail alerts Sign up to receive free email-alerts related to this article or journal.

Reprints and To order reprints of this article or to subscribe to the journal, contact the AACR Publications Subscriptions Department at pubs@aacr.org.

Permissions To request permission to re-use all or part of this article, contact the AACR Publications Department at permissions@aacr.org. 